\title{
Detailed Geant 4 simulations of the ANITA and ANITA-CUP neutron facilities
}

\author{
Q. Hong*, S. P. Platt*, A. V. Prokofiev ${ }^{\dagger \ddagger}$ and E. Passoth ${ }^{\ddagger}$ \\ * School of Engineering, University of Central Lancashire, England \\ $\dagger$ Division of Applied Nuclear Physics, Uppsala University, Sweden \\ $\ddagger$ The Svedberg Laboratory, Uppsala University, Sweden
}

\begin{abstract}
Simulations of the ANITA spallation neutron source at The Svedberg Laboratory (TSL) are described. Neutron radiation calculations show close agreement with measurements at both standard and close user positions. Gamma radiation characteristics are also predicted.
\end{abstract}

\section{INTRODUCTION}

The ANITA facility [1] at The Svedberg Laboratory (TSL) in Uppsala, Sweden, has been widely used for accelerated testing for neutron single-event effects (SEE). A high-flux upgrade of the facility, ANITA-CUP, was recently introduced and reported [2].

Platt et al. have reported preliminary Monte Carlo simulations of neutron and gamma production at the standard ANITA facility [3]. That work was motivated by a need to understand possible sensitivity to gamma rays in detectors used for beam monitoring during SEE tests. Simulations of a bare spallation target showed qualitative agreement with independent calculations and measurements of the fast neutron field [1]. The present work was motivated partly by a desire to investigate whether improvements to the geometry used in the simulations would lead to closer quantitative agreement with measurements, and partly by a need to extend the work to encompass the new ANITA-CUP facility [2].

Accordingly, in this paper we present high-fidelity analyses of neutron and gamma fields at ANITA and ANITA-CUP, with comparison to independent models and validation against measurements.

\section{Modelling}

Simulations were conducted using Geant4 version 10.0 [4], [5] with the binary intranuclear cascade model (see also [3]). A detailed model of the ANITA geometry was implemented in Geant4, as illustrated in Fig. 1; a summary only is given here. The spallation target is a tungsten cylinder of diameter $5 \mathrm{~cm}$ and length $2.4 \mathrm{~cm}$. The target is cooled by water and surrounded by a stainless-steel cooling jacket. The target assembly with its cooling jacket appears as a small blue rectangle in Fig. 1. Lead shielding blocks in the target region are also modelled, and shown in red Fig. 1. A large electromagnet surrounding the target is modelled and shown in Fig. 1 in grey (iron) and yellow (copper). An iron collimator with variable apertures is modelled and shown downstream of the target (grey). Simulated protons were incident at an energy of $180 \mathrm{MeV}$. Resulting neutron and gamma fields were

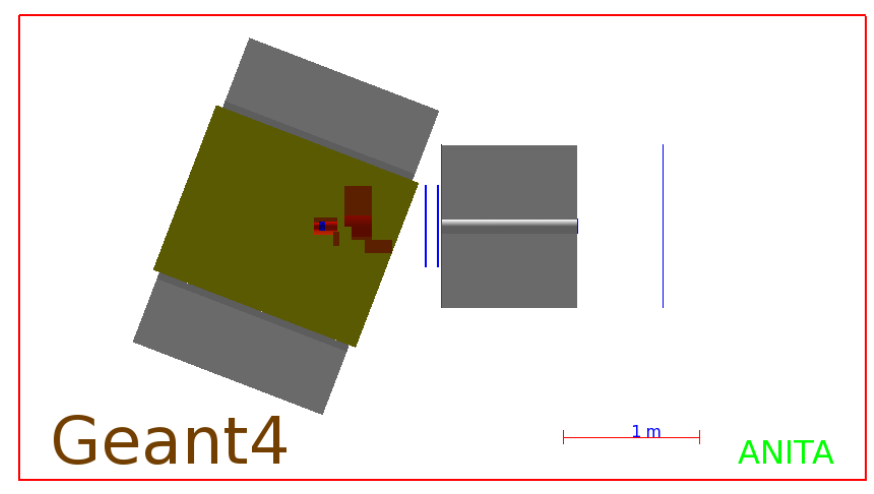

Fig. 1: Simulated ANITA facility overview seen from above (yellow: bending magnet; red: shielding components; blue: neutron production target assembly; grey: collimator; blue: virtual detector system)

evaluated at several virtual detector locations. In this paper results are presented for three of these: the Standard User Position (SUP), $2.5 \mathrm{~m}$ downstream of the target, the Close User Position (CUP), $0.75 \mathrm{~m}$ downstream of the target, and the CUP-TOF position, $0.84 \mathrm{~m}$ downstream of the target. The SUP position is downstream of a collimator with variable aperture; the CUP and CUP-TOF positions are upstream of the same collimator. The SUP and CUP positions are of interest as they are positions used for SEE tests. Thin-film breakdown counter (TFBC) detectors used for beam monitoring and characterisation including neutron time of flight (TOF) measurements are placed at the CUP-TOF position. TFBC data are used to validate simulation results. All the results presented here are for a primary proton current of $215 \mathrm{nA}$ and, with one exception (to match available experimental data), for the standard collimator aperture $(10.2 \mathrm{~cm}$ diameter $)$.

\section{RESULTS}

\section{A. Neutrons}

Fig. 2a shows neutron distribution at the SUP, for neutrons above $10 \mathrm{MeV}$. The collimator ensures that the beam is circular. Within the beam umbra the calculated neutron fluence rate above $10 \mathrm{MeV}$ is $7.05 \times 10^{5} \mathrm{n} \mathrm{cm}^{-2} \mathrm{~s}^{-1}$, compared to $6.38 \times 10^{5} \mathrm{n} \mathrm{cm}^{-2} \mathrm{~s}^{-1}$ from earlier calculations [3] and $1.0 \times 10^{6} \mathrm{n} \mathrm{cm}^{-2} \mathrm{~s}^{-1}$ from measurements [1]. The present calculations underestimate measurements at the SUP by $30 \%$. 


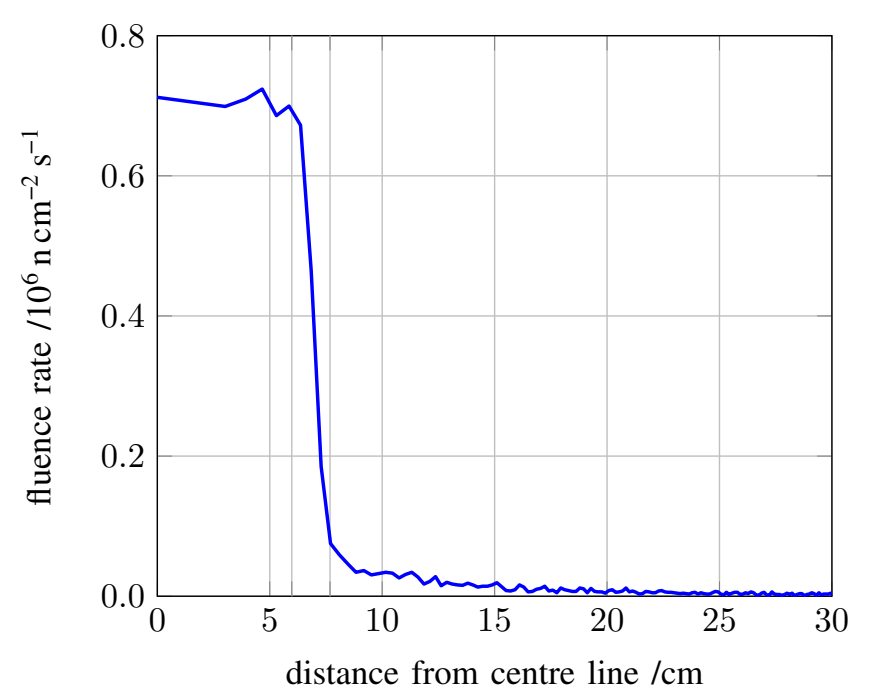

(a) Standard User Position. Nominal umbra and penumbra limits are also shown $(6.0 \mathrm{~cm}$ and $7.7 \mathrm{~cm}$, respectively).

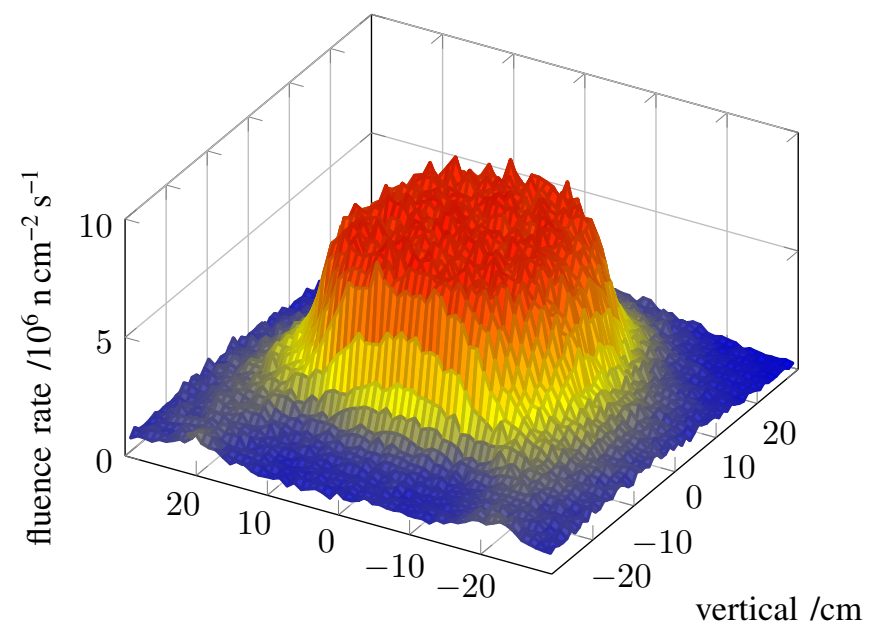

horizontal position $/ \mathrm{cm}$

(b) Close User Position

Fig. 2: Spatial distribution of neutrons above $10 \mathrm{MeV}$ at SUP and CUP

Fig. 2b shows the spatial distribution of neutrons above $10 \mathrm{MeV}$ at the CUP. As CUP is upstream of the collimator the distribution is not circularly symmetric. In the central region the calculated neutron fluence rate above $10 \mathrm{MeV}$ is $7.85 \times 10^{6} \mathrm{n} \mathrm{cm}^{-2} \mathrm{~s}^{-1}$, compared to $1.17 \times 10^{7} \mathrm{n} \mathrm{cm}^{-2} \mathrm{~s}^{-1}$ from measurements [2]. The present results underestimate measurements at the CUP by $33 \%$.

TFBCs equipped with ${ }^{238} \mathrm{U}$ targets were used to measure beam profile and time of flight spectra at the CUP-TOF position. Simulated neutron counts were folded in energy with the ${ }^{238} \mathrm{U}$ fission cross-section [6] and compared with measurements. Fig. 3 shows the resulting vertical profiles of the neutron-induced fission rate in ${ }^{238} \mathrm{U}$. The results are in general agreement although the simulation results are somewhat broader than measurements and the sharp cut-off beyond

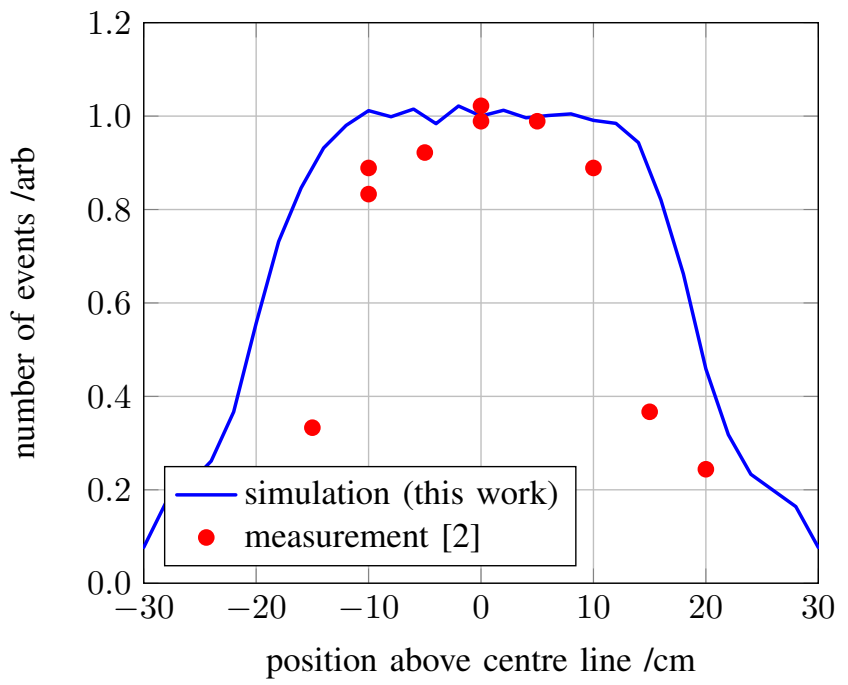

Fig. 3: Vertical profile of ${ }^{238} \mathrm{U}$ fission events at the CUP

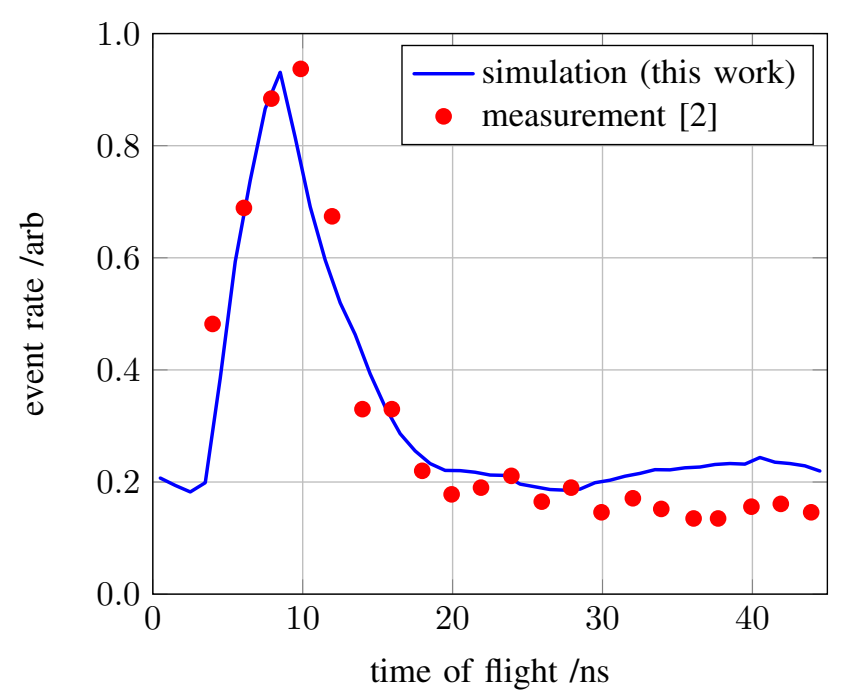

Fig. 4: Time of flight spectra for ${ }^{238} \mathrm{U}$ fission events at the CUP-TOF position, with $3 \mathrm{~cm}$ diameter collimator.

$11 \mathrm{~cm}$ is not fully reproduced by the simulation.

In Fig. 4 we compare simulated and measured TOF spectra at the CUP-TOF position. Neutron TOF data from the simulation were folded in energy with the ${ }^{238} \mathrm{U}$ fission cross-section, convolved with a $5 \mathrm{~ns}$ rectangle function approximating the primary proton micropulse shape, and overlapped at $45 \mathrm{~ns}$, representing the timing ambiguity due to the micropulse period. The results show very close agreement in the position and width of the TOF peak. The continuum beyond about $30 \mathrm{~ns}$, corresponding to neutrons below about $40 \mathrm{MeV}$, is somewhat higher in the calculation than in the measurement. This might indicate a relative overestimate of lower energy neutron flux in the calculations.

Fig. 5 compares calculated spectra at SUP and CUP with analytical fits based on MCNPX simulations and TOF measure- 


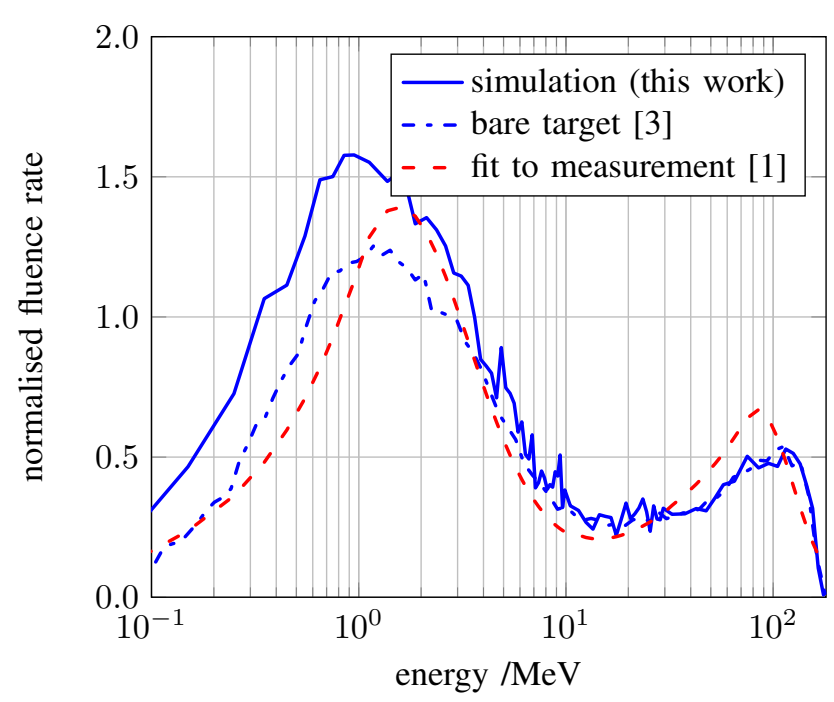

(a) SUP

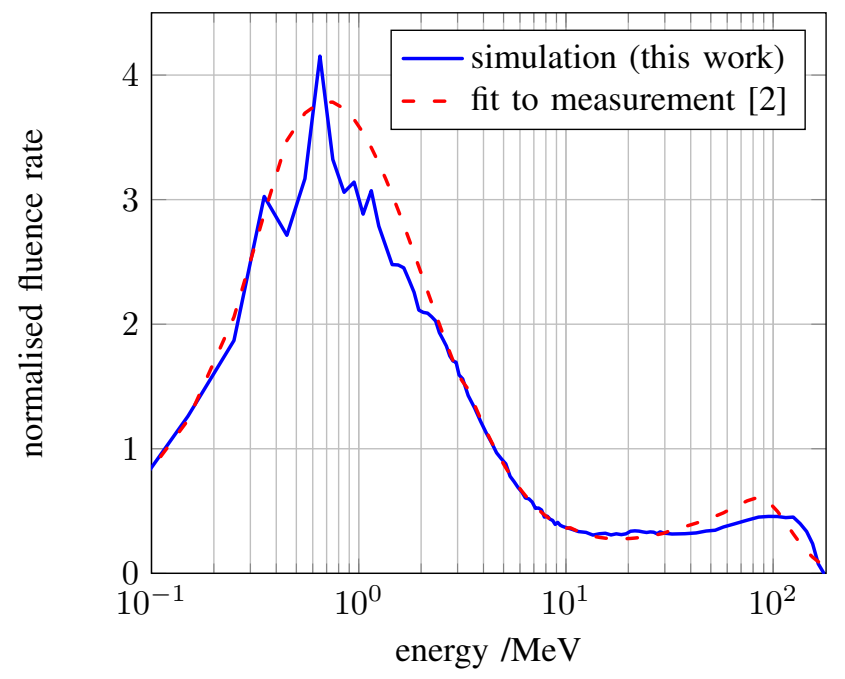

(b) CUP

Fig. 5: Calculated neutron spectra, normalised to fluence above $10 \mathrm{MeV}$

ments [1], [2]. The SUP data are also compared with results from Geant4 simulations of the bare target [3]. These curves are normalised to neutron fluence above $10 \mathrm{MeV}$ and presented as lethargy plots to emphasise the shape of the spectra rather than their integral fluence rate. The increased integral fluence rate at SUP from the present calculations, compared to the preliminary results of [3], is therefore not visible in Fig. 5a. At SUP, the present results show an increased contribution below about $1 \mathrm{MeV}$, consistent with interactions in materials other than the target, absent from the preliminary simulations. Fig. $5 \mathrm{~b}$ shows good agreement between the present results and the analytical fit from [2], especially in the evaporation peak around $1 \mathrm{MeV}$ (the fit cannot reproduce the structure there).

When compared to the fits the calculated spectra from the current work lead to slightly lower neutron yield and slightly

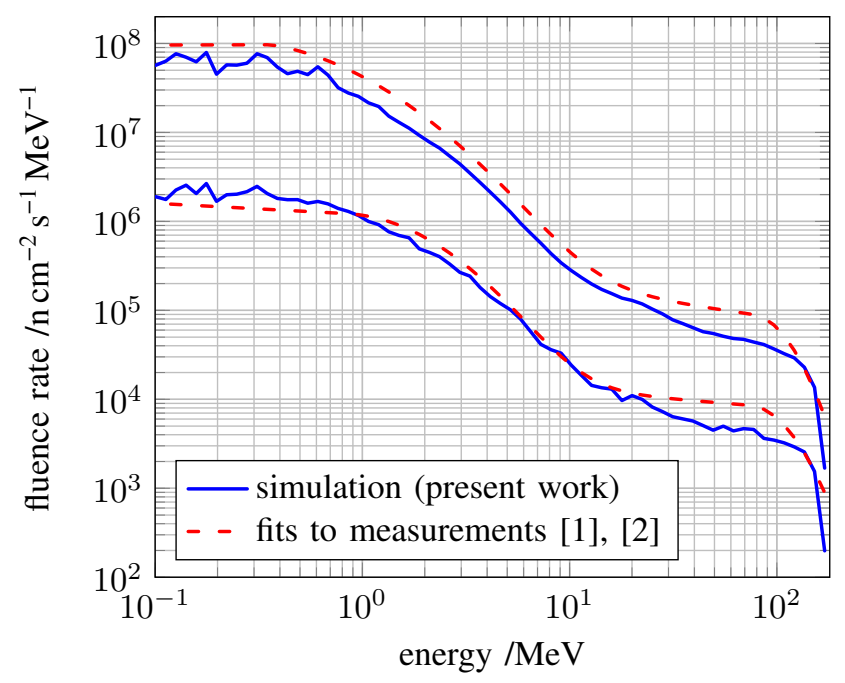

Fig. 6: Differential neutron spectra at CUP (upper curves) and SUP (lower curves)

higher peak energies in the higher energy peak near $100 \mathrm{MeV}$. This is consistent with results for the simulated TOF spectra (see Fig. 4 and associated discussion.) It is not surprising that this is unaffected by the improved model geometry as this peak arises from nucleon cascades in the target.

Fig. 6 summarises neutron spectra results by comparing differential flux as calculated in this work with the analytical fits. The SUP calculations and fit agree very closely in the range from $1 \mathrm{MeV}$ to $20 \mathrm{MeV}$; the fit exceeds the calculations somewhat above that energy. At CUP the fit exceeds the Geant4 calculations somewhat at all energies, slightly more so above $20 \mathrm{MeV}$.

\section{B. Photons}

Fig. 7 shows the calculated spatial distribution of gamma photons at SUP and CUP, and shows a gamma radiation distribution that is qualitatively very similar to that for neutrons at both locations. Fig. 8 shows the calculated differential gamma spectra within the central region of the spatial distribution. There is a broad continuum up to an energy of about $10 \mathrm{MeV}$, with a peak in the fluence spectrum at about $0.5 \mathrm{MeV}$ (where the annihilation peak is also visible). The gamma fluence rate is an order of magnitude greater at CUP that at SUP, consistent with the closer range to the target and its immediate surroundings. The CUP spectrum also shows a backscatter peak around $0.2 \mathrm{MeV}$.

Fig. 9 shows the calculated gamma dose spectrum, obtained by folding the gamma spectra (Fig 8) with dose conversion data from [7]. The results show structure with peaks around $1.5 \mathrm{MeV}, 3 \mathrm{MeV}, 6 \mathrm{MeV}$ and $9 \mathrm{MeV}$. Note that these are not coincidence peaks, as the simulation counts individual photons. The calculated dose rate is $20.9 \mathrm{mSv} \mathrm{h}^{-1}$ at SUP and $369 \mathrm{mSv} \mathrm{h}^{-1}$ at CUP. The calculated value at SUP is slightly more than and therefore consistent with that predicted from a bare spallation target $\left(17 \mathrm{mSv} \mathrm{h}^{-1}\right)$ and is also consistent with 


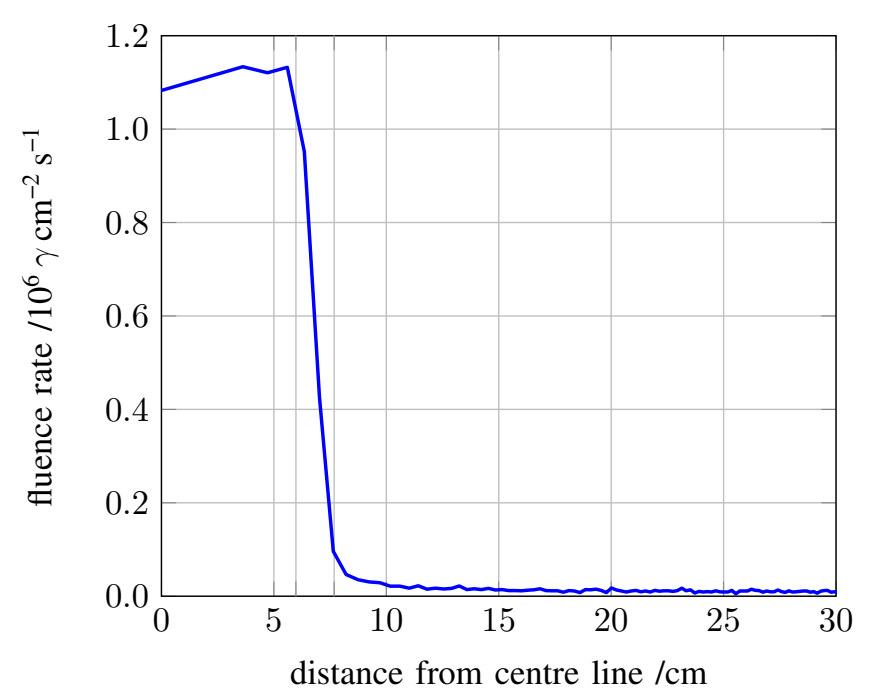

(a) Standard User Position. Nominal umbra and penumbra limits are also shown $(6.0 \mathrm{~cm}$ and $7.7 \mathrm{~cm}$, respectively).

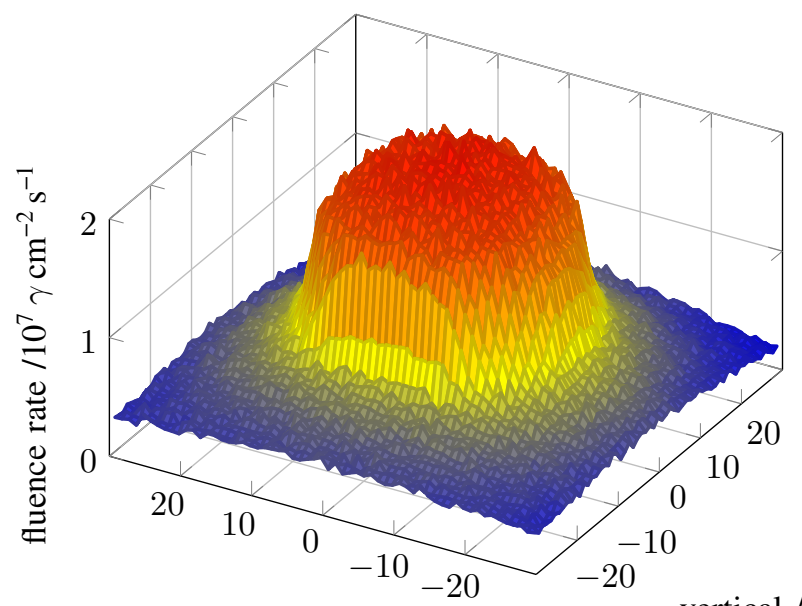

vertical $/ \mathrm{cm}$

horizontal position $/ \mathrm{cm}$

(b) Close User Position

Fig. 7: Calculated gamma spatial distribution

an estimated upper limit of $40 \mathrm{mSv} \mathrm{h}^{-1}$ reported in [1]. The calculated value at CUP is consistent with that calculated at SUP (a ratio of $18: 1$ is somewhat greater than $1 / R^{2}$, as would be expected), but rather more than the estimated upper limit $\left(170 \mathrm{mSv} \mathrm{h}^{-1}\right)$ given in [2].

\section{CONCLUSION}

We have used Geant 4 for detailed Monte Carlo simulations of the ANITA and ANITA-CUP neutron SEE test facilities at The Svedberg Laboratory. Neutron fluence rate, energy spectrum, time profile and spatial distribution show good agreement with measurements and independent simulations (using MCNPX). Gamma radiation characteristics have also been predicted and preliminary evaluations indicate that they are consistent with a limited set of measurement data. Evaluation of our results is continuing.

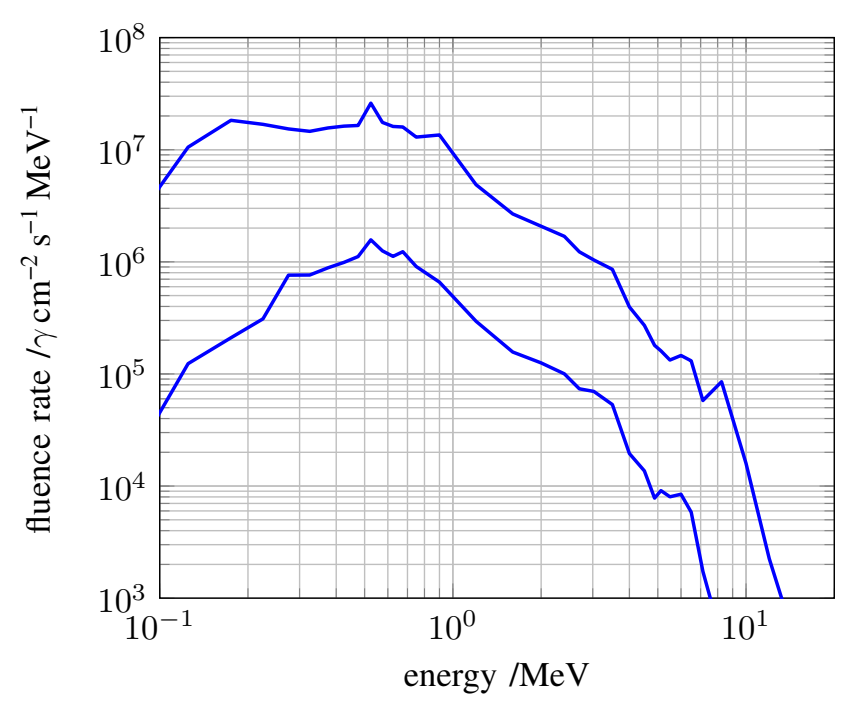

Fig. 8: Calculated gamma spectra at CUP (upper curve) and SUP (lower curve)

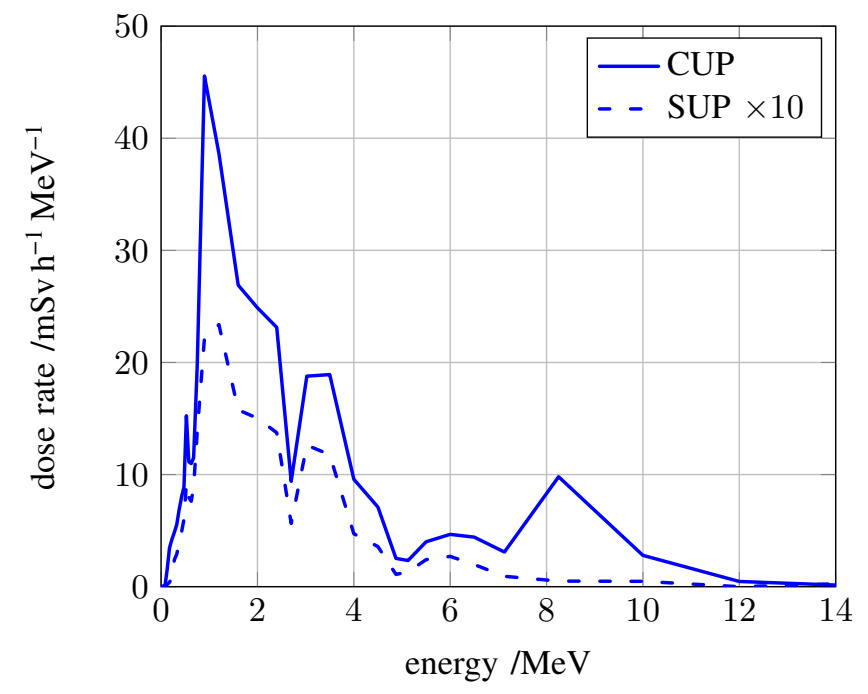

Fig. 9: Calculated gamma dose spectra

\section{REFERENCES}

[1] A.V. Prokofiev et al., "Characterization of the ANITA neutron source for accelerated SEE testing at The Svedberg Laboratory", 2009 IEEE Radiat. Effects Data Workshop Record, pp. 166-173

[2] A.V. Prokofiev et al., "CUP-A new high-flux irradiation position at the ANITA neutron facility at TSL", IEEE Trans. Nucl. Sci. vol. 61 no. 4, pp. 1929-1936, 2014

[3] S. P. Platt et al., "Neutron and gamma fields at neutron spallation sources for single-event-effects testing", Proc. 14th European Conf. Radiat. Effects Compon. Syst., 2013

[4] S. Agostinelli et al., "Geant4-a simulation toolkit", Nucl. Inst. Meth. Phys. Res. A vol. 506, pp. 250-303, 2003

[5] J. Allison et al., "Geant4 developments and applications", IEEE Trans. Nucl. Sci. vol. 53 no. 1, pp. 270-278, 2006

[6] A. D. Carlson et al., "International evaluation of neutron cross section standards", Nucl. Data Sheets vol. 110 no. 12, pp. 3215-3324, 2009

[7] S.-G. Kwon et al., "Calculation of neutron and gamma-ray flux-to-doserate conversion factors", J. Kor. Nucl. Soc. vol. 12 no. 3, pp. 171-179, 1980 